\title{
Visualization of Mechanical Loads with Semiconductor Nanocrystals
}

\author{
Martin Moebius ${ }^{1}$, Joerg Martin ${ }^{2 \star}$, Melinda Hartwig1 ${ }^{1}$, Ricardo Decker ${ }^{1}$, Lothar Kroll ${ }^{1,3}$, \\ Reinhard R. Baumann ${ }^{1,2}$, Thomas Otto ${ }^{1,2}$
}

\author{
${ }^{I}$ Technische Universität Chemnitz, Chemnitz 09126, Germany \\ ${ }^{2}$ Fraunhofer Institute for Electronic Nano Systems, Chemnitz 09126, Germany \\ ${ }^{3}$ Fraunhofer Institute for Machine Tools and Forming Technology, Chemnitz 09126, Germany
}

*Corresponding author: Tel: (+49) 37145001 244; E-mail: joerg.martin@enas.fraunhofer.de

Received: 14 August 2018, Revised: 18 October 2018 and Accepted: 13 December 2018

DOI: $10.5185 /$ amlett.2019.2221

www.vbripress.com/aml

\begin{abstract}
Fibre-reinforced plastics offer excellent mechanical properties at low weight. Hence, such materials are ideally suited to reduce energy consumption and $\mathrm{CO}_{2}$ emission, e.g. in aircraft and automotive engineering, shipbuilding or in the field of renewable energies. However, in contrast to e.g. metals, lightweight structures are sensitive to mechanical loads exceeding a certain approved range. In order to detect mechanical overloads at an early stage and to avoid consequential failures in lightweight structures, we recently proposed a novel concept of a thin-film sensor for visualization of mechanical loads by using photoluminescence quenching of quantum dots. Here, we present results according to the optimization of the ionization efficiency of the cadmium selenide quantum dots by using poly( $\mathrm{N}$-vinylkarbazol) (PVK) as charge transport material with favorable energy levels. Measurements of the photoluminescence intensity and electrical power confirm an increase of efficiency with almost the same photoluminescence drop compared to $\mathrm{N}, \mathrm{N}, \mathrm{N}^{\prime}, \mathrm{N}^{\prime}$-Tetrakis(3-methylphenyl)3,3'-dimethyl-benzidine (HMTPD), most likely by the higher valence band offset between quantum dots and PVK. Furthermore, an integration of a layer stack with connected ceramic piezoelectric transducer demonstrates the successful use of the sensor system for mechanical load detection in lightweight structures. Copyright $\odot$ VBRI Press.
\end{abstract}

Keywords: Structural health monitoring, lightweight structures, photoluminescence, quantum dots.

\section{Introduction}

Since the first investigations in 1981 by Alexey Ekimov and the formation of the term quantum dots (QDs) by Mark Reed in 1988, the use of semiconductor colloidal nanoparticles has increased in various fields of applications [1, 2]. Due to the small band photoluminescence (PL) of QDs, the nanoparticles are used especially in display technology to improve color gamut [3]. However, further research in other fields of application is still ongoing. In solar cells, for example, due to the absorption of a larger range of the solar spectrum a higher energy yield can be achieved [4]. Also in electronic devices such as field effect transistors (FETs), as biomarkers in medicine or for visualization of carbon nanotubes, QDs are used [5-7].

Due to the nano-size of QDs, the nanoparticles are outstandingly suited for film-based sensors and actuators. Processing on flexible substrates, such as polyethylene terephthalate (PET) foils, a large variety of customized shapes is possible. This leads to a major application area for integration in lightweight structures by resin infusion, laminating or injection molding. Additionally, a large-scale integration of sensors and actuators already during the manufacturing processes generates additionally cost advantages. Thereby our focus is on the development of film-based sensors for mechanical load detection.

By the increasing use of lightweight structures for the reduction of energy consumption especially in aerospace, automotive engineering, shipbuilding and renewable energies, the monitoring of these lightweight structures is of increasing importance. Especially fiberreinforced plastics are sensitive to abrupt mechanical loads. Related microscopic small defects within the material are difficult to detect with naked eye and might lead to spontaneous failures. The result is a destruction of the entire lightweight structure and associated consequential costs.

Therefore, an autarkic sensor, which is able to detect mechanical loads on lightweight structures by optical visualization, could help to identify possible defects in the lightweight structure at an early stage and to prevent total failure during operation. The general working principle of our sensor is based on the influence of the photoluminescence of quantum dots by electrical charge carriers [8-10]. The film-based sensor consists of semiconducting organic materials with embedded QDs 
and a piezoelectric layer. However, an independent spatial arrangement of both parts is also possible. In initial state, incident UV light is absorbed and visible PL light is emitted (Fig. 1 left).

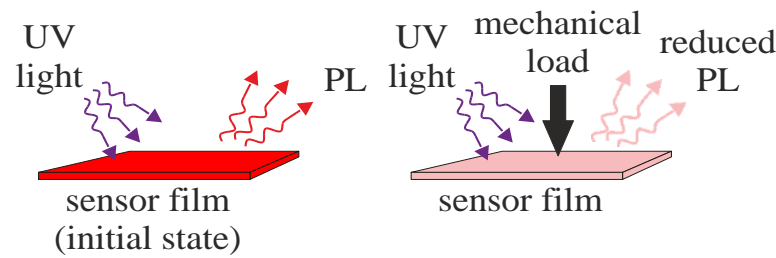

Fig. 1. PL intensity before (left) and after mechanical load (right).

If a mechanical load is acting, the piezoelectric layer generates charge carriers. These charge carriers reach the quantum dots via the electrodes and charge transport layers and reduce their PL intensity. (Fig. 1 right). The strength of the mechanical load influences the PL differently and thus forms an indication for the strength of a possible damage.

Therefore, different requirements exist for the sensor, such as high optical contrast and high initial PL intensity as well as high efficiency for an optimal utilization of generated charge carriers produced by the piezoelectric element. Compared to previous works, we use a layer stack with improved efficiency and quantum dots with higher quantum yield. Nevertheless, the efficiency must be further improved to realize an autarkic mechanical load detection on lightweight structures $[\mathbf{9}, \mathbf{1 1}]$. Therefore, we compare layer stacks with two different organic charge transport materials to optimize the efficiency of the sensor film. Furthermore, by integration of layer stacks with connected ceramic piezoelectric transducers in fibre-reinforced plates, we demonstrate the functionality of the sensor system.

\section{Experimental}

The schematic representation in Fig. 2 shows the structure of the two-layer stacks with different organic materials.
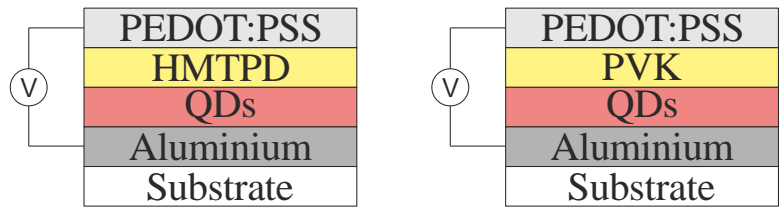

Fig. 2. Layer stack with HMTPD (left) and PVK (right).

For both layer stacks we use $100 \mathrm{~nm}$ thick aluminum electrodes (anode) evaporated trough a shadow mask onto $100 \mu \mathrm{m}$ thick PET substrates. The substrates are cleaned before in acetone, ethanol and deionized water for 15 minutes, respectively, in an ultrasonic bath. Afterwards CdSe/CdS QDs (CANdot Series A plus) with a quantum yield above $60 \%$ for good visibility of QDs PL are spin coated from a hexane dispersion with a concentration of $2.5 \mathrm{mg} / \mathrm{ml}$ directly on top of the aluminum electrode. Subsequently, we applied two different organic materials to allow a comparison of the
PL intensity behavior and the efficiency of the layer stacks. For one layer stack, a $40 \mathrm{~nm}$ thick $\mathrm{N}, \mathrm{N}, \mathrm{N}^{\prime}$, $\mathrm{N}^{\prime}$-Tetrakis (3-methylphenyl)-3, 3'-dimethyl-benzidine (HMTPD, Sigma Aldrich) layer is deposited by a thermal evaporation process directly onto the QD layer (Fig. 2 left) [8]. For the comparative layer stack, a layer of poly(N-vinylkarbazol) (PVK, Sigma Aldrich) with a thickness of approx. $70 \mathrm{~nm}$ was spin coated on top of the QDs (Fig. 2 right). To finalize the both layer stacks, a $50 \mathrm{~nm}$ transparent electrode consisting of poly (3, 4-ethylenedioxy-thiophene) -poly (styrenesulfonate) (PEDOT:PSS, Orgacon IJ-1005) is deposited on top of the HMTPD and PVK layer by inkjet printing (Fujifilm, DMP 2831).

In order to characterize the functionality of both layer stacks, we use a self-build confocal micro spectroscopy setup with attached grating monochromator (Andor Shamrock SR-303i-A), which allows a recording of time-resolved series of PL-spectra. Thereby a solid-state laser module (B\&WTek Inc.) with a wavelength at $475 \mathrm{~nm}$ excites the QDs photoluminescence. Finally, each individual spectrum is integrated and normalized to the initial PL intensity.

To investigate the influence of the electric charge carriers on the PL of the QDs a voltage is applied to the layer stacks simultaneously to PL recording, whereby the PEDOT:PSS electrode is grounded and the positive polarity is applied to the aluminum electrode. The cross sectional area of aluminum and PEDOT:PSS is $1 \mathrm{~mm}^{2}$ for each measurement.

In addition, we have integrated the layer stacks in fibre-reinforced structures by using vacuum infusion technology to prove the functionality after integration. To demonstrate the functionality as a closed sensors system, we also integrated ceramic piezoelectric transducers.

As depicted in Fig. 3, individual layers of the laminate, the layer stack and the piezoelectric transducer are placed on a solid mold cavity and covered with a polymeric film.

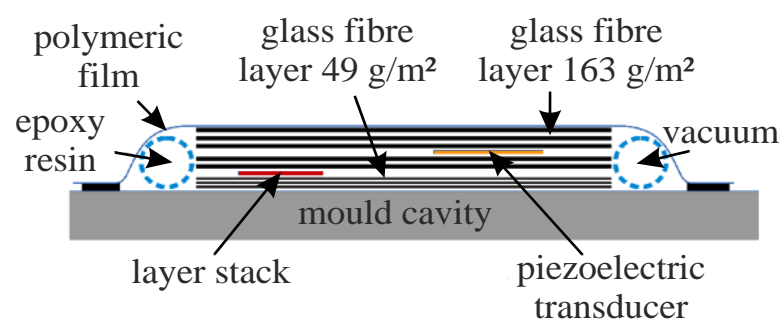

Fig. 3. Schematic view of integrating layer stack and piezoelectric transducer by vacuum infusion technology.

The laminate consists of eight layers of flat woven glass fibre fabrics with a fibre orientation of $0^{\circ}$ and $90^{\circ}$. In order to position the layer stacks close to the surface of the fibre-reinforced structure, a lower grammage of $49 \mathrm{~g} / \mathrm{m}^{2}$ for the first three layers of glass fibre fabrics is used. Thereby, the layer stacks are located between the third and the fourth layer. Each of the upper five layers have a grammage of $163 \mathrm{~g} / \mathrm{m}^{2}$ to achieve a certain 
thickness and the required mechanical properties of the fibre-reinforced structure. The piezoelectric transducer is located between the fifth and the sixth layer and thereby connected to the sensor layer stack via thin copper wires. Due to the difference in pressure between vacuum and environment, evacuation of the layers takes place and a thermoset plastic is infused. As matrix material, the epoxy resin L with the hardener GL 2 (R\&G Faserverbundwerkstoffe $\mathrm{GmbH}$ ) is used. Curing the matrix material finalizes the fibre-reinforced structure.

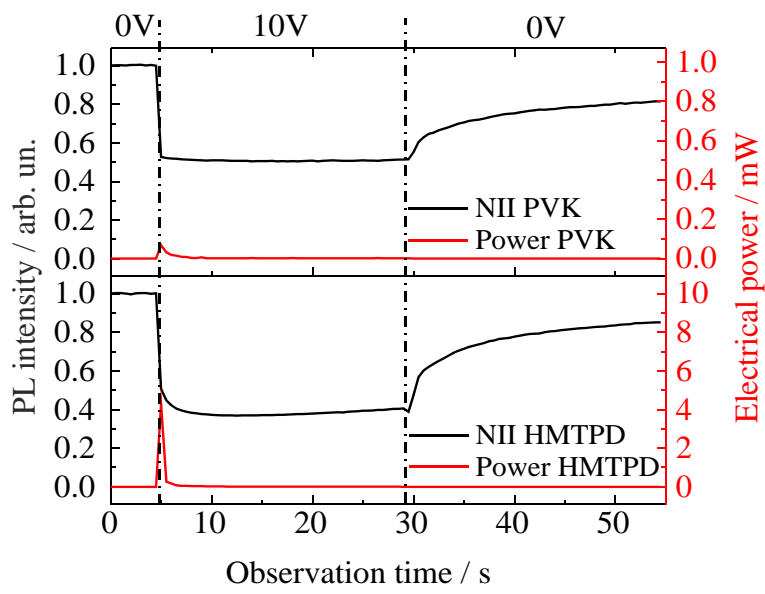

Fig. 4. Photoluminescence and electrical power of layer stack with PVK and HMTPD.

\section{Results and discussion}

Fig. 4 shows the PL intensity as well as the electric power of the layer stack with PVK and HMTPD within an observation time of $55 \mathrm{~s}$. By applying a voltage of $10 \mathrm{~V}$ after five seconds of recording to both layer stacks, a fast drop in PL intensity occurs.

For PL quenching via an Auger process, an additional non-localized charge must be present within the quantum dot [12]. This charge can be either injected into the nanoparticle or generated via photoinduced ionization. For HMTPD we already assign the dominant process for reducing the PL intensity to an electron ejection out of the QD core [8].

Indeed, the energy levels of PVK are very similar to HMTPD (Fig. 5). The small offset of $0.1 \mathrm{eV}$ for conduction band and $0.2 \mathrm{eV}$ for valence band suggest an assignment of PVK to the same processes for PL reduction as described for HTMPD $[\mathbf{1 3}, \mathbf{1 4}]$.

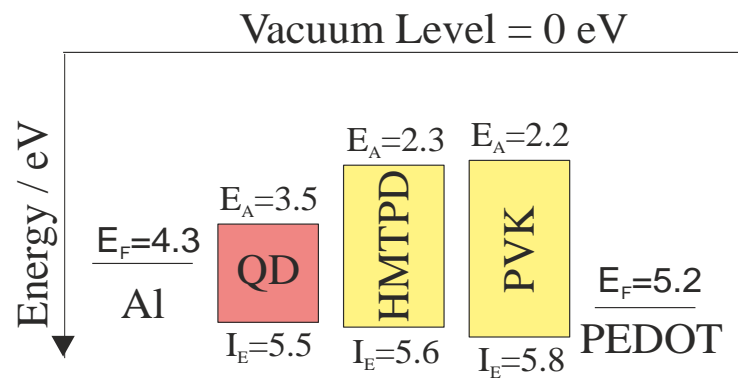

Fig. 5. Energy level diagram of layer stack with HMTPD and PVK.
However, the difference is that the PL intensity drops to approx. $50 \%$ of initial PL intensity in case of PVK instead of $40 \%$ in case of HMTPD. A possible cause could be that the solvent of the PVK solution dissolves a part of the QD layer, whereby some QDs embedded in PVK via a phase separation process [15]. As a result, the electron ejection rate to the aluminum electrode is reduced and thus more QDs remain in the bright state. It is important to know that the layer stack with PVK has a lower initial PL intensity, which also indicates a dissolving of the QDs layer during the spin coating process of PVK. Regarding the large conduction band offset between QDs and the adjacent organic materials, there is a low probability for electron tunneling processes. As a result, an exchange of electrons between PEDOT:PSS and the QD core is very unlikely and therefore the influence of these charge carriers on the PL reduction mechanism seems to be very low.

However, the slightly larger valence band offset between PVK and QDs compared to HTMPD and QDs is more significant. This results in a higher barrier for holes to reach the valence band of PVK. As a consequence the electrical power has a lower short maximum of approx. $70 \mu \mathrm{W}$ compared to $4 \mathrm{~mW}$ in case of HMTPD and decreases afterwards to $500 \mathrm{nW}$ instead of $5 \mu \mathrm{W}$ in case of HMTPD before the voltage was turned off. The initial current drop we assign most likely to a chemical reduction of PEDOT polymer chains by electron injection into the transparent electrode [16]. In addition, also other factors seem to influence the sensor stack. The higher thickness of PVK with approx. $70 \mathrm{~nm}$ compared to approx. $40 \mathrm{~nm}$ in case of HMTPD and also the lower hole mobility of PVK with $4.8 \cdot 10^{-6} \mathrm{~cm}^{2} / \mathrm{Vs}$ compared to HMTPD with $2.5 \cdot 10^{-3} \mathrm{~cm}^{2} / \mathrm{Vs}$ also limit the current flow and thus the electrical power in the sensor stack $[17,18]$.

For both layer stacks, a fast increase followed by a slower increase in PL intensity characterizes the PL restoring behavior. The similarity between the curvatures suggests the same processes for both organic materials, e.g. charge tunneling, as already described in $[8]$.

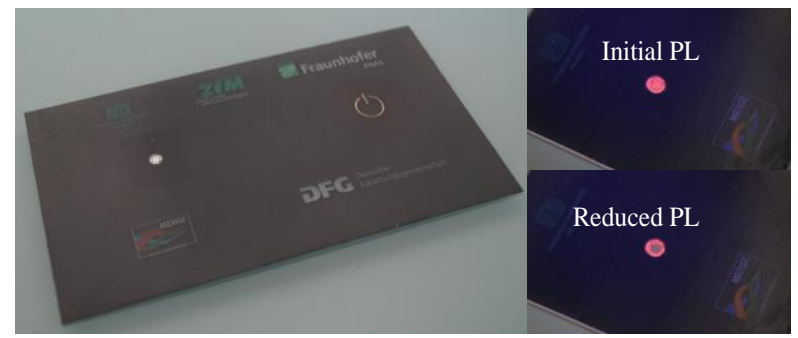

Fig. 6. Fibre-reinforced plastic plates with integrated layer stack and ceramic piezoelectric transducer.

In order to demonstrate the functionality of the mechanical load detection sensor, we have manufactured fibre-reinforced plastic plates with integrated layer stacks and ceramic piezoelectric transducers. As depicted in Fig. 6, a mechanical load on the integrated 
ceramic piezoelectric transducer reduces the PL intensity in the designated circular area with a diameter of $3 \mathrm{~mm}$. This test shows that it is possible to cause reduction in the PL intensity by a very small amount of charge carriers in the range of micro coulomb produced by the piezo-electric transducer.

\section{Conclusion}

In this paper, we described the structure of a layer stack in combination with a ceramic piezoelectric transducer, which is able to detect and visualize mechanical loads in lightweight structures. To optimize the efficiency of the layer stack, we tested PVK as organic material in comparison to HMTPD. It was apparent, that the higher valence band offset between QDs and PVK and higher layer thickness enable a more efficient layer stack with almost the same PL drop. Additionally, a successful integration of the layer stack with connected ceramic piezoelectric transducer in fibrereinforced plates demonstrates the possible use of the sensor system in lightweight structures for mechanical load detection.

\section{Acknowledgements}

The work was performed within the Federal Cluster of Excellence EXC 1075 "MERGE Technologies for Multifunctional Lightweight Structures" and supported by the German Research Foundation (DFG). Financial support is gratefully acknowledged.

\section{References}

1. Ekimov, A. I.; Onushchenko, A. A., Jetp Lett., 1981, 34, 345.

2. Reed, M. A.; Randall, J. N.; Aggarwal, R. J.; Matyi, R. J.; Moore, T. M.; Wetsel, A. E., Phys. Rev. Lett., 1988, 60, 535.

3. Virey, E., Reports on Phosphors and Quantum Dots, Yole Developpement, 2015

4. Chuang, C. H. M.; Brown, P. R.; Bulović, V.; Bawendi, M. G., Nat. Mater., 2014, 13, 796.

5. Hetsch, F.; Zhao, N.; Kershaw, S. V.; Rogach, A. L., Mater. Today, 2013, 16, 312.

6. Clark, D.; Pazdernik, N., Molekulare Biotechnologie: Grundlagen und Anwendungen; Springer Verlag: Germany, 2009.

7. Chaudhary, S.; Kim, J. H.; Singh, K. V.; Ozkan, M., Nano Lett., 2004, 4, 2415.

8. Moebius, M.; Martin, J.; Hartwig, M.; Baumann, R. R.; Otto, T.; Gessner, T., AIP Adv., 2016, 6, 085309.

9. Moebius, M.; Ma, X.; Martin, J.; Doty, M. F.; Otto, T.; Gessner, T., Quantum Sensing and Nanophotonic Devices XII, Proc. of SPIE, San Francisco, 2015, 9370.

10. Weiss, A.; Moebius, M.; Martin, J.; Otto, T.; Smart Systems Integration for Micro- and Nanotechnologies, Chapter Semiconductor Nanocrystals - Modern Building Blocks for Innovative Microsystems, Goldenbogen, Germany, 2014.

11. Martin, J.; Staudinger, U.; Demir, E.; Spudat, C.; Pötschke, P.; Voit, B.; Otto, T.; Gessner, T., Integrated Optics: Devices, Materials, and Technologies XVI, Proc. of SPIE, 2012, 8264.

12. Chepic, D. I.; Efros, Al. L.; Ekimov, A. I.; Ivanov, M. G.; Kharchenko, V. A.; Kudriavtsev, I. A.; Yazeva, T. V., J. Lumines., 1990, 47, 113.

13. Lee, S. J.; Park, J. S.; Song, M.; Yoon, K. J.; Kim, Y. I.; Jin, S. H.; Seo, H. J., Appl. Phys. Lett., 2008, 92, 193312.

14. Adachi, C.; Baldo, M. A.; Thompson, M. E.; Forrest, S. R., J. Appl. Phys., 2001, 90, 5048.

15. Coe-Sullivan, S.; Steckel, J. S.; Woo, W. K.; Bawendi, M. G.; Bulović, V., Adv. Funct. Mater., 2005, 15, 1117.

16. Ha, H.; Kim, O., Appl. Phys. Lett., 2008, 93, 033309.
17. D’Angelo, P.; Barra, M.; Cassinese, A.; Maglione, M. G.; Vacca, P.; Minarini, C.; Rubino, A., Solid-State Electron., 2007, 51, 123.

18. Barito, A.; Sykes, M. E.; Huang, B.; Bilby, D.; Frieberg, B.; Kim, J.; Green, P. F.; Shtein, M., Adv. Energy Mater., 2014, 4, 1. 